\title{
Estudo da prevalência de fluorose dentária em Aracaju
}

\author{
Study of the prevalence of dental fluorosis in Aracaju
}

\author{
Ricardo Wathson Feitosa de Carvalho ${ }^{1}$ \\ Roberta Barreto VieiraValois ${ }^{2}$ \\ Cléa Núbia Albuquerque Santos ${ }^{3}$ \\ Paulo Sérgio M arcellini ${ }^{4}$ \\ Leonardo Rigoldi Bonjardim 5 \\ Cristiane Costa da Cunha O Iiveira ${ }^{2}$ \\ Sandra Regina Barretto ${ }^{2}$ \\ Suzane Rodrigues ) acinto Gonçalves ${ }^{1}$
}

Abstract With the aims of determining the prevalence dental fluorosis in the city of Aracaju, SergipeState, 196 students weresubmitted to an oral exam, utilizing the Dean's index. It was concluded that the prevalence of dental fluorosis in students ranging from 5 to 15 years old in the city of Aracaju, Sergipe State was of $8.16 \%$, not implying in a risk to public health. However, similar studies must be done regularly, besides orientation to governmental departments responsible for water fluoridation, so that the level considered excellent for this area can be respected, avoiding such problem to occur.

Key words Dental fluorosis, Prevalence, Oral health
Resumo Com o objetivo de determinar a prevalência de fluorose dentária em Aracaju (SE), 196 escolaresforam submetidosa exameclínico bucal, utilizando o índice de Dean. Pôde ser concluído que a prevalência de fluorose dentária em escolares de cinco a quinze anos do município de Aracaju (SE) foi de 8,16\%, não implicando risco à saúde pública. Porém, estudos semel hantes devem ser realizados com periodicidade regular, além de orientação aos órgãos responsáveis pela fluoretação da água, para que os níveis considerados ótimos para essa região sejam respeitados, prevenindo assim o surgimento de tal ocorrência.

Palavras-chave Fluorose dentária, Prevalência, Saúde bucal

\footnotetext{
${ }^{1}$ Faculdade de $\mathrm{O}$ dontologia, Universidade de Pernambuco. Avenida General N ewton Cavalcanti 1650, Tabatinga. 54753-220 Camaragibe PE watshon@ig.com.br

2 UniversidadeTiradentes.

${ }^{3}$ USF Edivaldo Correia Barbosa.

${ }^{4}$ Universidade Federal de Sergipe.

${ }^{5}$ Departamento de Fisiologia, Centro de Ciências Biológicas e da Saúde, Universidade Federal deSergipe.
} 
Introdução

Um dos marcos mais importantes da história da odontologia foi a descoberta das propriedades anticariogênicas dos fluoretos, pois possibilitou o desenvolvimento de medidas eficazes de prevenção e controle da cárie dentária ${ }^{1}$. 0 maior responsável pelo declínio da cárie dentária nas últimas décadas foi o flúor, aplicado à população através da água fluoretada, esquema terapêutico coadjuvado por outras técnicas de aplicação, como os dentifrícios fluoretados? ${ }^{2}$.

Porém, o uso de fluoretos, sistêmico ou tópico, no tratamento ou prevenção de cárie dentária pode resultar na ingestão e absorção do mesmo para a circulação sanguínea. Assim, a mineralização dos dentes em formação pode ser afetada, acarretando fluorose dentária ${ }^{3}$.

Os sinais clínicos da fluorose dentária são caracterizados por linhas brancas finas que seguem as periquimáticas do esmalte ou até um aspecto total menteopaco ecal cário. A pós a erupção, nas formas mais graves, pode ocorrer o desprendimento de porções do esmalte, levando ao aparecimento de depressões da superfície do dente. Outra característica dessa patologia é a existência de certa simetria no grau em que os dentes homólogos são afetados ${ }^{4}$.

Entretanto, 0 aumento de fluorose não deve trazer consigo um desestímulo ao uso deste elemento em programas de prevenção econtrole da cárie dentária, uma vez que seu uso é comprovadamente um dos responsáveis pelo declínio do índice de cárie amplamente observado nas últimas décadas ${ }^{5}$. M edidas de vigilância sanitária devem ser recomendadas às autoridades responsáveis, pois a manutenção das bases de sistemas de prevenção assegura o máximo de benefício, com redução do índice de cárie e o mínimo de risco de fluorose dentária 6 .

No município de Aracaju (SE), $88,18 \%$ da população é abastecida pela Companhia de Saneamento Básico de Sergipe, na qual a fluoretação das águas teve início em 19807.

Assim, é importante que a concentração de flúor utilizada em águas de abastecimento público seja cautelosamente monitorada, minimizando riscos decorrentes do uso abusivo desse halogênio. 0 município deAracaju (SE) carecedeinformações oficiais acerca de fluorose dentária; portanto, o objetivo do presente trabalho foi obter dados de prevalência dessa patologia em escolares das EMEIS, de cinco a quinze anos, buscando subsídios para divulgar a importância do uso racional do flúor como instrumento nos programas preventivos de cárie dentária.
M etodologia

Para este trabalho, foi utilizada uma amostra probabilística em múltiplo estágio, com unidades amostrais primárias de escolas públicas e particulares da cidade deAracaju (SE). Para cada distrito territorial, foi realizado um sorteio para escolha do estabel ecimento de ensino, perfazendo assim quinze escolas a serem visitadas. Foram selecionados aleatoriamente e examinados 196 escolares (cinco a quinze anos) de ambos os gêneros, matriculados em escolas da rede municipal de ensino de Aracaju (SE).

0 treinamento ea calibração do examinador se deram por meio de um estudo de diapositivos de indivíduos com graus variados de fluorose e por exames visuais das arcadas dentárias de crianças portadoras dessa alteração.

Ao primeiro contato com os pais das crianças, estes leram e assinaram o termo de consentimento livre eesclarecido, aprovado pelo ComitêdeÉtica da Universi dadeTiradentes. Em seguida, as crianças foram submetidas ao exame clínico, realizado em cadeira comum sob luz natural ecampo seco obtido com o auxílio de gaze. A presença e 0 grau de fluorose em cada unidade dentária presentena cavidadeoral de cada criança foram pesquisados através da utilização do índice de Dean, proposto pela OM S, no qual 0 grau de fluorose varia de zero (normal) a cinco (severo), conforme abaixo (Tabela 1).

Os dados de cada criança foram anotados em ficha clínica, especialmente desenvolvida para essa pesquisa, sendo tabulados e analisados através do programa M icrosoft Office Excel 2007, desenvolvido pela Microsoft Corporation.

\section{Resultados}

Após tabulação e análise estatística dos dados, os seguintes resultados foram observados:

- a prevalência defluorose dentária no município de Aracaju (SE), ou seja, de crianças que apresentaram fluorose dentária do grau 2 (muito leve) ao 5 (severo), foi de 8,16\% (16 casos) (Figura 1);

0 grau de zero (normal) de fluorose foi encontrado em 141 (71,94\%) crianças, o grau 1 (questionável) em 39 (19,9\%), o grau 2 (muito leve) em 14 (7,14\%), o grau 3 (leve) em 1 (0,51\%), o grau 4 (moderado) em $0(0,0 \%)$ e o 5 (severo) em 01 (0,51\%) (Figura 1).

- A faixa etária variou de cinco a quinze anos, com maior número, 73 crianças (37,24\%), entre 
Tabela 1. Índice de Dean, proposto pela OMS.

$$
\text { Índice de Dean }
$$

$\begin{array}{lcl}\begin{array}{l}\text { Normal } \\ \text { Questionável }\end{array} & 0 & \begin{array}{l}\text { Esmalte superficial liso, brilhante e geralmente de cor branca bege pálida. } \\ \text { Esmalte apresenta leves aberrações na translucidez de esmalte normal, que podem } \\ \text { variar desde pequenos traços esbranquiçados até manchas ocasionais. }\end{array} \\ \text { Muito leve } & 2 & \begin{array}{l}\text { Áreas pequenas e opacas de cor branca, porosas e dispersas irregularmente sobre o } \\ \text { dente, mas envolvendo menos de } 25 \% \text { da superfície dentária vestibular. } \\ \text { Opacidade branca do esmalte mais extensa do que para o código 2, recobrindo } \\ \text { Leve }\end{array} \\ \text { Moderado } & 4 & \begin{array}{l}\text { Superfície de esmalte apresentando desgaste acentuado e manchas marrons, } \\ \text { frequentemente alterando a anatomia do dente. } \\ \text { Superfície do esmalte muito afetada e hipoplasia tão acentuada que o formato geral } \\ \text { do dente pode ser afetado. Existem áreas com fóssulas ou desgastes e manchas } \\ \text { marrons espalhadas por toda parte; os dentes frequentemente apresentam aparência } \\ \text { de corrosão. }\end{array}\end{array}$

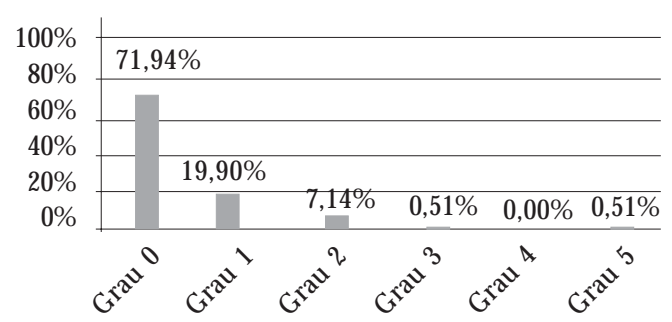

Figura 1. Distribuição dos escolares de cinco a quinze anos de escolas da rede municipal de ensino de Aracaju (SE), segundo o índice de fluorose de Dean $(n=196)$.

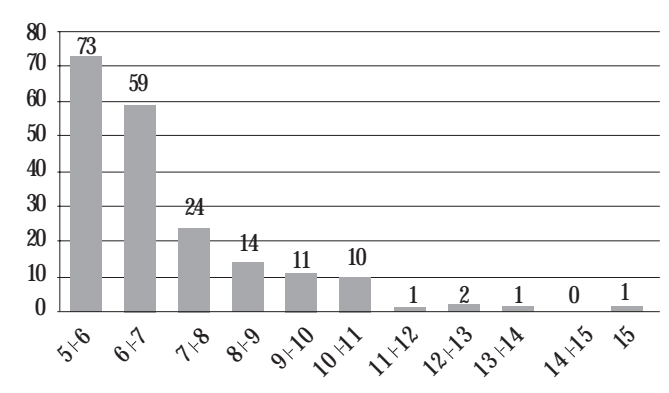

Figura 2. Distribuição dos escolares de cinco a quinze anos de escolas da rede municipal de ensino de Aracaju (SE), segundo faixa etária $(n=196)$.

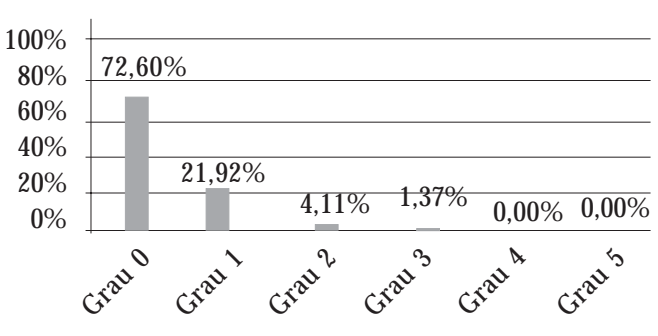

Figura 3. Distribuição dos escolares de cinco a seis anos de escolas da rede municipal de ensino de Aracaju (SE), segundo o índice de fluorose de Dean $(n=73)$.

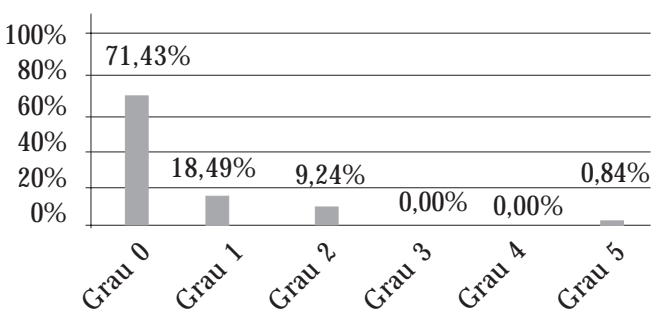

Figura 4. Distribuição dos escolares de seis a doze anos de escolas da rede municipal de ensino de Aracaju (SE), segundo o índice de fluorose de Dean $(n=119)$. 


\section{Discussão}

Quando se fala em fluorose dentária, a maioria dos estudos é realizada nas regiões sul, sudestee centro-oestedo país. Embora exista fonte deágua para consumo humano com teor elevado deflúor nas regiões norte e nordeste, poucos estudos foram realizados4.

A cáriedentária ainda éo principal problema de saúde bucal a ser solucionado, sendo o uso do flúor uma medida eficaz na sua prevenção: À medida que se observa a redução do índice de cárie, também se verifica aumento da prevalência de fluorose $e^{9,10}$.

Quando utilizado em níveis racionais ideais, o flúor tem efeito benéfico na prevenção e controle da cárie dentária ${ }^{11}$. Porém, quando utilizado de forma crônica e excessiva, duranteo período de formação dos dentes, pode causar manchas no esmalte, conhecidas como fluorose ${ }^{3}$.

A fluoretação das águas de abastecimento entre as regiões do Brasil é muito discrepante. No nordeste, estima-se que apenas $12,36 \%$ da população seja coberta; no sul, 93,09\%, norte, centro-oestee sudeste, $12,98 \%, 49,11 \%$ e $91,92 \%$, respectivamente ${ }^{12}$. No Estado de Sergipe, a fluoretação da água de abastecimento público teve início em 19807.

A fluoretação da água de abastecimento público, ainda que seja considerada uma medida necessária para o controle da cárie, tem se somado à difusão maciça de outras formas de flúor, contribuindo para o aumento da prevalência de fluorose ${ }^{11}$.

As pesquisas quanto ao nível ideal de flúor na áqua de abastecimento tiveram início com Dean, em 1931, que afirmou que o mesmo deveria ser entre 1,0 e 1,5 ppmF ${ }^{13}$. A concentração de flúor na água de abastecimento considerada ótima é $0,7 p p m F$. 0 teor de flúor varia de acordo com a temperatura da região ${ }^{14}$. No Estado de São Paulo, o teor ideal é de $0,7 \mathrm{mg} / \mathrm{L}$, podendo variar entre 0,6 a 0,8 mg/L ${ }^{15}$. Em Sergipe, a faixa devalor considerada aceitável éde 0,6 a 1,0ppmF?.

A fluorose dentária é caracterizada pelo surgimento de manchas opacas, sendo o diagnóstico diferencial fundamental para o estabel ecimento do plano de tratamento, que vai desde uma microabrasão do esmalte à confecção de facetas de resina composta ${ }^{16}$. 0 diagnóstico diferencial deveser feito com opacidades não induzidas pelo flúor; lesão demancha branca incipiente da cárie; esmalte hipoplásico, dentinogênese imperfeita e manchas por tetraciclina ${ }^{13}$. A realização de tratamento somente é necessária nos casos mais gra- ves, nos quais a fluorose se torna um problema cosmético ${ }^{17}$.

$\mathrm{N}$ a atualidade, no Brasil, o único método sistêmico preventivo dedoença cárieéa fluoretação da água de abastecimento público ${ }^{18}$. Esseéconsiderado o mais econômico e abrangente método deprevenção de cárie ${ }^{19}$. No município deAracaju (SE) , 88,18\% da população é abastecida pela Companhia de Saneamento Básico de Sergipe?.

No estudo realizado por Trendley Dean nas décadas de trinta equarenta, em regiões naturalmente fluoretadas, demonstra-se que o máximo de benefício na prevenção da cárie é alcançado com a presença de sinais de fluorose muito leve em uma pequena proporção (10 a 15\%) da comunidade ${ }^{6}$.

A pesar dos diferentes índices utilizados para registrar fluorose dentária, recentes estudos observaram quea prevalência de fluorose em diversas zonas urbanizadas do país está abaixo de $30 \%$ para cidades com 0,8 ppmF na água de consumo ${ }^{19-22}$. U ma prevalência acima deste percentual tem sido atribuída ao efeito combinado de produtos dentários e fluoretos em águas de abastecimento ${ }^{23}$. A fluorose dentária na Paraíba varia em torno de $31 \%$ em áreas com 0,4 a 0,7ppm, 61\% em áreas com 0,8 a 1,0ppm e 71\% em áreas com fluoreto em águas deconsumo acima de 1,0 ppm ${ }^{24}$.

Dentre os estudos de prevalência de fluorose dentária publicados no Brasil, utilizando o índice de Dean, é destacado o de Barros et al..$^{25}$, que examinaram 159 escolares de doze anos de idade em Ouro Preto (M G) e relataram prevalência de $11,4 \%$, sen do composta pela condição muito leve $(10,1 \%)$ e leve (1,3\%). Em nosso estudo, em crianças com doze anos de idade, o índice de fluorose foi de $0 \%$; porém, a representatividade dessa idade foi muito pequena, sendo representada por apenas dois sujeitos, ou seja, $1,02 \%$ do total.

No município deSanta Tereza, Rio Grandedo Sul, Toassi e Abegg ${ }^{11}$ analisaram 259 escolares de quatro a dezoito anos deidade, encontrando prevalência de $63,7 \%$ de fluorose dentária, sendo o grau muito leve predominante $(43,6 \%)$, seguido pelo grau leve $(12,0 \%)$, moderado $(7,7 \%)$, questionável $(7,3 \%)$ e severo $(0,4 \%)$. Na faixa etária de quatro a dezoito anos deidade, a presentepesquisa avaliou 260 menores ( $70,46 \%$ do total) e a distribuição de fluorose foi $47,43 \%$ grau 0 (sem fluorose), 15,45\% grau 1 (questionável), 6,23 grau 2 (muito leve), $0,81 \%$ grau 3 (leve), $0,27 \%$ graus 4 e 5 (moderado e severo, respectivamente).

Cangussu et al. ${ }^{26}$, em Salvador, estudaram 3.313 adolescentes de doze equinzeanos deidade, tendo como resultado prevalência de $31,4 \%$ nos 
escolares de doze anos e 27,6\% aos quinze anos, predominando a categoria "muito leve". Na presente pesquisa, na referida faixa etária, foi encontrado $1,53 \%$ grau 0 e $0,51 \%$ grau 1 , porém com apenas quatro representantes ( $2,04 \%$ do total).

No município de Aracaju (SE), a prevalência defluorose, considerando a faixa etária de cinco a quinze anosfoi de $8,16 \%$, ou seja, de crianças que apresentaram fluorose dentária do grau 2 (muito leve) ao 5 (severo). Porém, como se pode ver pelas considerações acima, foi considerada a importância de se estratificar as faixas etárias para pode obter dados que fornecessem parâmetros consistentes de comparação. Assim, a prevalência de fluorose foi avaliada considerando tal estratificação, ou seja, pré-escolares ( 5 a 6 anos; $n=73$ ) e escolares ( 6 a 12 anos; $n=119$ ). A faixa etária acima de doze anos foi descartada por possuir pe quena representatividade na amostra total $(n=4)$.

M esmo assim, a prevalência de fluorose foi baixa, ou seja, a faixa etária de cinco a seis anos foi de $5,48 \%$, sendo $4,11 \%$ muito leve $(n=3)$ e $1,37 \%$ leve $(n=1)$. Na faixa etária de seis a doze anos, a prevalência encontrada foi de $10,08 \%$, sendo $9,24 \%$ muito leve $(n=11)$ e $0,84 \%$ severo $(n=1)$.

O levantamento epidemiológico possui relevante importância em nível de planejamento e execução dos serviços odontológicos ${ }^{27}$. As medidas desaúde pública deimpacto populacional são efetivas, porém, existe uma parcela da população que necessita de cuidados adicionais ${ }^{17}$. 0 reconhecimento da realidadeepidemiológicaédefundamental importância para o planejamento dos programas de saúde bucal ${ }^{28}$. Estudos devem ser feitos periodicamente para monitorar os níveis e os padrões da alteração ${ }^{6}$. As capacitações dos cirurgiões-dentistas no serviço público devem incluir o assunto fluorose dentária e vigilância.

Em 25 de agosto de 2005, foi proposto o projeto de lei no 29729, em substituição à atual normatização legal ${ }^{30}$, na qual consta, in verbis, $[. .$. art. 1o, parágrafo único. A utilização de flúor na profilaxia da cárie dentária só pode ser realizada pela aplicação tópica do elemento, ficando proibida a adição deflúor ou qualquer deseus compostos a água, bebidas ou alimentos. Portanto, constituindo a tese de quea ação profilática na prevenção à cárie ocasiona o surgimento defluorose, efeitos neurológicos colaterais, infertilidadeehipotireoidismo. Porém, a lei que é regulamentada no Brasil ainda éa que dispõe a fluoretação da água de abastecimento público.

Não é a proibição da fluoretação das águas de abastecimento públicos a solução para a prevenção dos efeitos deletérios do flúor e, sim, me didas de vigilância sanitária sobre produtos fluoretados ${ }^{6}$. A proibição da fluoretação de água sob a al egação de que essa medida é a causa de problema e a não produção de conhecimentos mais consistentes pode significar prejuízo para a saúde coletiva. Estudos revelam que, em locais onde houve tal proibição, a taxa de cárie se elevou $^{31,32}$. Tais decisões não se justificam, sendo juridicamenteilegais, cientificamenteinsustentáveis e socialmente injustas ${ }^{33}$.

\section{Conclusão}

Podemos concluir, pelo exposto, que a prevalência de fluorose dentária em crianças de cinco a quinze anos de idade do município de Aracaju (SE), ou seja, que apresentaram fluorose dentária do grau 2 (muito leve) ao 5 (severo), foi de8,16\%, não implicando risco à saúde pública. Porém, estudos semelhantes devem ser realizados com periodicidade regular, buscando determinar se existem outros fatores de risco associados, além de orientação aos órgãos responsáveis pela fluoretação da água, para que os níveis considerados ótimos para essa região sejam respeitados, prevenindo assim o surgimento de tal ocorrência.

\section{Colaboradores}

RWF Carvalho, RBV Valois, CNA Santos e SRJ Gonçalves trabalharam na pesquisa de campo, metodologia e redação do artigo. CCC Oliveira, PS M arcellini, LR Bonjardim, SR Barretto e SRJ Gonçalves trabalharam na concepção, delineamento e análise e interpretação dos dados, bem como na revisão crítica do artigo. Todos participaram igualmente da aprovação da redação final do artigo.

\section{Agradecimentos}

0 presente trabalho foi realizado com 0 apoio do Conselho Nacional de Desenvolvimento Científico e Tecnológico - CN Pq. 


\section{Referências}

1. Oliveira BH, Milbourne P. Fluorose dentária em incisivos superiores permanentes em crianças de escola pública do Rio de Janeiro. Rev. Saude Publica 2001; 35(3):276-282.

2. Scabar LF, Armonia PL, Nicolau T, Barros FC, M ello [A]. O creme dental fluoretado (500 ppm F-) e o risco de fluorose dentária. Rev Inst Ciênc Saúde 2004; 22(4):305-309.

3. Fejerskov O, Manji F, Baleum V. The nature and mechanisms of dental fluorosis in man. J Dent Res 1990; 69:692-700.

4. Fejerskov O, Baelum V, Manji F, Moller IJ. Fluorose dentária: um manual para profissionais de saúde. São Paulo: Editora Santos; 1994

5. Pires M BO. Fluorose dentária endêmica: revisão da literatura. Unimontes Cientif 2002; 2(2):1-15.

6. Frazão P, Peverari AC, Forni TIB, M ota G, Costa LR. Fluorose dentária: comparação de dois estudos de prevalência. Cad Saude Publica 2004; 20(4):1050-1058.

7. Companhia de Abastecimento de Sergipe. Sergipe 2007. [favor completar a referência com o nome da publicação, documento, etc.]

8. Gomes PR, Costa SC, Cypriano S, Sousa LR. Paulínia, São Paulo, Brasil: situação da cárie dentária com relação às metas OM S 2000 e 2010. Cad Saude Publica 2004; 20(3):866-870.

9. Lima YBO, Cury JA. Ingestão de flúor por crianças pela água e dentifrícios. Rev. Saude Publica 2001 35(6):576-581.

10. Paiva $S$ M, Cury JA. Dentifrício fluoretado e risco de fluorose dentária. RPG Rev Pós Grad 2001; 8(4):322-328.

11. Toassi RFC, Abegg C. Fluorose dentária em escolares de um município da serra gaúcha, Rio Grande do Sul, Brasil. Cad Saude Publica 2005; 21(2):625-655.

12. Brasil. Ministério da Saúde. Fluoretação da água de consumo público - Parecer técnico-científico da saúde bucal. Brasília: Ministério da Saúde; 1996.

13. Burt BA. Fifty years of water fluoridation. $\mathrm{Br}$ Dent 1995; 178(2):49-50.

14. M elo JH. Os cones de saturação nos sistemas simplificados de fluoretação de áqua de abastecimento público [dissertação]. Brasília (DF): Associação Brasileira de Odontologia do Distrito Federal; 2004

15. Companhia de Saneamento Básico do Estado de São Paulo. Controle Sanitário. São Paulo: Sabesp; 2006.

16. Pinheiro IVA, M edeiros M C, Andrade AKM, Ruiz PA. Lesões brancas no esmalte dentário: como diferenciá-las e tratá-las. RBPO 2003; 2(1):11-18.

17. Silva $B B, M$ altz $M$. Prevalência de cárie, gengivite e fluorose em escolares de 12 anos de Porto Alegre RS, Brasil. Pesqui O dontol Bras 2001; 15(3):208-214.

18. Alves NC, Orenha ES, Reche NSG, Pelli MPS. Pre valência de fluorose dentária na dentição decídua em crianças participantes de programas de prevenção e promoção de saúde no município de $M$ arília-SP. Rev Fac Odontol Bauru 2002; 10(3):156-163.

19. Campos DL, Farias DG, Toledo AO, Bezerra ACB. Prevalência de fluorose dentária em escolares de Brasília - Distrito Federal. Rev Odontol Univ São Paulo 1998; 12(3):225-30.
20. Alcântara CM. Prevalência de fluorose dental em escolares de Curitiba. Rev ABO Nac 1998; 6(5):304307.

21. M altz M, Silva BB. Relationship of caries, gingivitis and fluorosis to socioeconomic status in Brazilian 12-year-old schoolchildren. Caries Res 2000; 34:315316.

22. Thylstrup A, Fejerskov O. Clinical appearance of dental fluorosis in permanent teeth in relation to histological changes. Community Dent Oral Epidemiol 1978; 6(6):315-328.

23. Valois CA, Soviero VM, Cruz RA. Avaliação do grau de fluorose dental em crianças de 7 a 12 anos de idade. J Bras Odontopediatr Odontol Bebê 1999; 2(9):383-390

24. Sampaio FC, Von Der Fehr FR, Arneberg P, Gigante DP, Hatloy A. Dental fluorosis and nutritional status of 6- to 11-year-old children living in rural areas of Paraíba, Brazil. Caries Rev 1999; 33:66-73.

25. Barros SFB, M atos DL. Prevalência de fluorose dentária em escolares de 12 anos de idade, Ouro Preto/ M G. Rev. bras. epidemiol. 2003; 8(4):425-431.

26. Cangussu MCT, Fernandez RAC, Rivas CCR, Ferreira C, Santos LCS. A fluorose dentária no Brasil: uma revisão crítica. Cad Saude Publica 2004; 18(1):7-15.

27. Pinto VG. Saúde bucal coletiva. 4a ed. São Paulo: Santos; 2000

28. Bardal PAP, Olympio KPK, Buzalaf AR, Bastos JRM . Dental caries and dental fluorosis in 7-12-year-old schoolchildren in Catalão, Goiás, Brasil. J Appl Oral Sci 2005; 13(1):1-7.

29. Brasil. Projeto de lei do senado no 297, de 2005. Determina que a utilização de flúor na profilaxia da cárie dentária só pode ser realizada pela aplicação tópica do elemento e proíbe a adição de flúor na água, bebidas e alimentos. Diário do Senado $\mathrm{Fe}$ deral 2005; 25 ago.

30. Brasil. Lei $n^{\circ} 6.050$ de 24 de maio de 1974. Dispõe sobre a fluoretação da água em sistemas de abastecimento quando existir estação de tratamento. Diário Oficial da União 1974; 24 mai.

31. Kunzel W. Effect of an interruption in water fluoridation on the caries prevalence of the primary and secondary dentition. Caries Res 1980; 14(5):304-310.

32. Stephen KW, McCall DR, Tullis JI. Caries prevalence in northern Scotland before and 5 years after water defluoridation. Br Dent J 1987; 163:324-326.

33. Narvai PC. Cárie dentária e flúor: uma relação do século XX. Cien Saude Colet 2000; 5(2):381-392.

Artigo apresentado em 06/07/2007

Aprovado em 14/01/2008

Versão final apresentada em 23/12/2008 